\title{
Formulation of Effective Chemical Management Strategy against Cercospora Leaf Spot Disease of Chilli
}

\author{
Dharnendra Reang", S. Khalko and A. Roy \\ Department of Plant Pathology, Faculty of Agriculture, Uttar banga Krishi Viswavidyalaya, \\ Pundibari, Coochbehar-736165, India \\ *Corresponding author
}

\begin{abstract}
A B S T R A C T
Field experiment was conducted during 2015 and 2016 to find out effective chemical management strategy against one most common disease of Chilli, which occurred throughout the year, i.e., Cercospora leaf spot. Among the six different fungicides treatments tested for spraying. All the treatments showed significantly different results than check. Lowest disease severity was found in Pyraclostrobin + Tebuconazole (Double application) (7.85\%). The highest percent disease control was observed with Pyraclostrobin + Tebuconazole (Double application) showing 64.69\% disease control followed by Pyraclostrobin + Tebuconazole (Single application) (61\%) and Azoxystrobin + Tebuconazole (Double application) $(57.98 \%)$. The treatment Azoxystrobin + Tebuconazole (Single application) also showed good control over Cercospora leaf spot of Chilli. Considering the yield, highest yield was obtained under Pyraclostrobin + Tebuconazole (Double application) with the estimated production of $1650.55 \mathrm{Kg} / \mathrm{ha}$ followed by Pyraclostrobin + Tebuconazole (Single application) and Azoxystrobin + Tebuconazole (Double application) with the production of $1285 \mathrm{Kg} / \mathrm{ha}$ and $1080 \mathrm{Kg} / \mathrm{ha}$, respectively. Among the treatments, Chlorothalonil (Double application) showed lowest disease control as well as lowest yield.
\end{abstract}

\section{Introduction}

Chilli (Capsicum annuum L.) or red pepper, a member of solanaceae is an important economic crop worldwide (Poulos, 1992). It is a remunerative vegetable, spice cum cash crop of the Indian subcontinent.

Among the known five Capsicum spp., Capsicum annuum is cultivated worldwide (Tong and Boseland, 1999) followed by Capsicum frutescens (Boseland and Votaven, 2003)
Chilli is mainly grown in India, Mexico, Japan, Indonesia, Pakistan and China. India ranks second in area and production after China. Andhra Pradesh is the leading chilli producing state contributing to about 58.0 per cent of the country's total production.

Karnataka is the second largest chilli producer $(13.0 \%)$ followed by Orissa $(4.8 \%)$, West Bengal (4.8 \%), Maharashtra (3.6\%) and Gujarat (3.5\%). During 2011, India account 40 per cent of the total chilli area in the world, which is about 7.92 lakh hectares with an 
annual production of $12.231 \mathrm{ac}$ MT of ripe dry chillies (Anonymous, 2012).

Chilli is used as a spice, condiment culinary supplement, medicine, vegetable and as an ornamental plant. Chillies are a good source of vitamin A, B, C (Ascorbic acid) and E (Tocopheral), oleoresin, carbohydrate and minerals such as calcium, phosphorus, ferrous, sodium and copper in trace amounts and the allied pungent principles viz., dihydrocapsaicin and nordihydrocapsaicin.

Chilli suffers from many diseases caused by fungi, bacteria, viruses, nematodes and also abiotic stresses. Among the fungal diseases, anthracnose or fruit rot, leaf spots, damping off and powdery mildew are the most prevalent ones.

\section{Materials and Methods}

An experiment field trial was conducted in randomized block design for consecutive two years 2015-2016 at Instructional Farm, UBKV, Pundibari to study the effect of application of different fungicides for controlling Cercospora leaf spot of chilli. Observations on the severity of Cercospora leaf spot was recorded starting from 30 DAT at an interval of 7 days.

The severity of Cercospora leaf spot was recorded on five randomly selected plants using a disease rating scale 0 to 9 scale was followed as suggested by McKinney (1923). Further, these ratings were converted to per cent disease index (PDI).

Further, theses scales were converted to per cent disease index (PDI) using the formula given by Mckinney (1923).

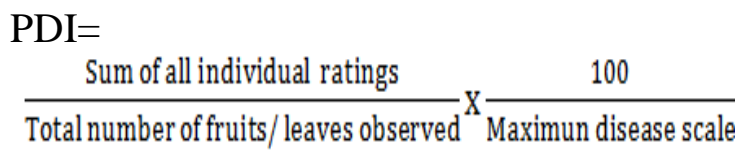

Six treatments were imposed besides a treatment with unsprayed control. The details of the treatments are furnished in Table 1.

\section{Results and Discussion}

The perusal of data in Table 2 indicates that lowest disease severity $(7.85 \%)$ was found in $\mathrm{T}_{4}$ treatment which is significantly lower than check. $\mathrm{T}_{4}$ showed lowest disease severity $(7.85 \%)$ and highest disease reduction $(64.69 \%)$ as compared to other treatments and also gave highest yield $(1650.55 \mathrm{~kg} / \mathrm{ha})$.

This was followed by $T_{3}, T_{2}$ and $T_{1}$ also gave good result both in terms of percent disease reduction as well as yield. The disease severity was $8.67 \%, 9.34 \%$ and $9.93 \%$ and disease reduction were $61.00 \%, 57.98 \%$ and $55.33 \%$ respectively. The yield were 1285, 1080 and $947 \mathrm{~kg} / \mathrm{ha}$ respectively.

Highest disease severity was found in $T_{7}$ followed by $\mathrm{T}_{6}$ and $\mathrm{T}_{5}$, the disease severity were $22.23 \%, \quad 14.15 \%$ and $10.89 \%$ respectively.

The disease reduction of $\mathrm{T}_{6}$ and $\mathrm{T}_{5}$ were $36.35 \%$ and $51.01 \%$ respectively and the yield were 736.40 and $888.62 \mathrm{~kg} / \mathrm{ha}$.

Thus, the pooled results of fungicidal trial of 2015 and 2016 clearly indicates the $T_{4}$ [Pyraclostrobin + Tebuconazole (Double application)] is the best treatment followed by $\mathrm{T}_{3}$ [Pyraclostrobin + Tebuconazole (Single application)] and $\mathrm{T}_{2}$ [Azoxystrobin + Difenconazole (Double application)] in reducing disease severity and increasing yield.

Similar work done by Kahn et al., (2005) who used Azoxystrobin and Tebuconazole along with some other fungicides for efficacy of Ccercospora leaf spot and found that a single, early application of Tebuconazole greatly reduced Cercospora leaf spot (Fig. 1 and 2). 
Table.1 Treatment spray schedule

\begin{tabular}{|c|c|c|c|c|}
\hline $\begin{array}{l}\text { Sl. } \\
\text { no. }\end{array}$ & Treatments & Treatment details & $\begin{array}{c}\text { Doses } \\
\text { (per litre) }\end{array}$ & $\begin{array}{l}\text { Spray } \\
\text { schedule }\end{array}$ \\
\hline 1 & $\mathrm{~T}_{1}$ & $\begin{array}{l}\text { Azoxystrobin }+ \text { Difenconazole } \\
\text { (Single application) }\end{array}$ & @ 1 ml & $\begin{array}{l}7 \text { days } \\
\text { interval }\end{array}$ \\
\hline 2 & $\mathrm{~T}_{2}$ & $\begin{array}{l}\text { Azoxystrobin + Difenconazole } \\
\text { (Double application) }\end{array}$ & @ $1 \mathrm{ml}$ & $\begin{array}{l}7 \text { days } \\
\text { interval }\end{array}$ \\
\hline 3 & $\mathrm{~T}_{3}$ & $\begin{array}{l}\text { Pyraclostrobin }+ \text { Tebuconazole } \\
\text { (Single application) }\end{array}$ & @ $1 \mathrm{ml}$ & $\begin{array}{l}7 \text { days } \\
\text { interval }\end{array}$ \\
\hline 4 & $\mathrm{~T}_{4}$ & $\begin{array}{l}\text { Pyraclostrobin + Tebuconazole } \\
\text { (Double application) }\end{array}$ & @ $1 \mathrm{ml}$ & $\begin{array}{l}7 \text { days } \\
\text { interval }\end{array}$ \\
\hline 5 & $\mathrm{~T}_{5}$ & Carbendazim (Double application) & (a) 1gm & $\begin{array}{l}7 \text { days } \\
\text { interval }\end{array}$ \\
\hline 6 & $\mathrm{~T}_{6}$ & Chlorothalonil (Double application) & @ $1.5 \mathrm{gm}$ & $\begin{array}{l}7 \text { days } \\
\text { interval }\end{array}$ \\
\hline 7 & $\mathrm{~T}_{7}$ & Control & - & - \\
\hline
\end{tabular}

Yield of the crop recorded at harvest and the data were statistically analyzed.

Table.2 Disease severity of Cercospora leaf spot (Pooled)

\begin{tabular}{|c|c|c|c|c|c|c|}
\hline \multirow[t]{2}{*}{ Treatments } & \multicolumn{3}{|c|}{ PDI } & \multirow[b]{2}{*}{$\begin{array}{l}\text { Disease } \\
\text { control } \\
\text { over } \\
\text { check } \\
(\%)\end{array}$} & \multirow{2}{*}{$\begin{array}{l}\text { Yield } \\
\text { (g/plot) }\end{array}$} & \multirow{2}{*}{$\begin{array}{l}\text { Estimated } \\
\text { yield } \\
\text { (kg/ha) }\end{array}$} \\
\hline & $\begin{array}{l}1^{\text {st }} \\
\text { Observa } \\
\text { tion }\end{array}$ & $\begin{array}{l}2^{\text {nd }} \\
\text { Observa } \\
\text { tion }\end{array}$ & $\begin{array}{l}3^{\text {rd }} \\
\text { Observa } \\
\text { tion }\end{array}$ & & & \\
\hline $\begin{array}{l}\mathrm{T}_{1} \text { [Azoxystrobin + Difenconazole } \\
\text { (Single application)] }\end{array}$ & $\begin{array}{l}1.56 \\
(1.41) * b\end{array}$ & $\begin{array}{l}6.74 \\
(2.69) d\end{array}$ & $\begin{array}{l}9.93 \\
(3.21) \mathrm{cd}\end{array}$ & 55.33 & 568.33 & 947.22 \\
\hline $\begin{array}{l}\mathrm{T}_{2} \text { [Azoxystrobin + Difenconazole } \\
\text { (Double application)] }\end{array}$ & $\begin{array}{l}2.82 \\
(1.80) a\end{array}$ & $\begin{array}{l}5.37 \\
(2.41) e\end{array}$ & $\begin{array}{l}9.34 \\
(3.11) c d\end{array}$ & 57.98 & 648 & 1080.00 \\
\hline $\begin{array}{l}\mathrm{T}_{3} \text { [Pyraclostrobin + } \\
\text { Tebuconazole (Single } \\
\text { application)] }\end{array}$ & $\begin{array}{l}1.93 \\
(1.54) a b\end{array}$ & $\begin{array}{l}4.67 \\
(2.27) f\end{array}$ & $\begin{array}{l}8.67 \\
(3.01) c d\end{array}$ & 61.00 & 771 & 1285.00 \\
\hline $\begin{array}{l}\mathrm{T}_{4} \text { [Pyraclostrobin }+ \\
\text { Tebuconazole (Double } \\
\text { application)] }\end{array}$ & $\begin{array}{l}2.35 \\
(1.71) a b\end{array}$ & $\begin{array}{l}4.22 \\
(2.17) \mathrm{g}\end{array}$ & $\begin{array}{l}7.85 \\
(2.86) d\end{array}$ & 64.69 & 990.33 & 1650.55 \\
\hline $\begin{array}{l}\mathrm{T}_{5} \text { [Carbendazim (Double } \\
\text { application)] }\end{array}$ & $\begin{array}{l}2.44 \\
(1.73) \mathrm{ab}\end{array}$ & $\begin{array}{l}7.56 \\
(2.84) c\end{array}$ & $\begin{array}{l}10.89 \\
(3.36) c\end{array}$ & 51.01 & 533.17 & 888.62 \\
\hline $\begin{array}{l}\text { T}_{6}[\text { Chlorothalonil (Double } \\
\text { application)] }\end{array}$ & $\begin{array}{l}2.38 \\
(1.54) a b\end{array}$ & $\begin{array}{l}8.74 \\
(3.03) b\end{array}$ & $\begin{array}{l}14.15 \\
(3.82) b\end{array}$ & 36.35 & 441.84 & 736.40 \\
\hline $\begin{array}{l}\text { T } \\
\text { [Control] } \\
\end{array}$ & $\begin{array}{l}1.63 \\
(1.55) \mathrm{ab}\end{array}$ & $\begin{array}{l}13.40 \\
(3.73) a\end{array}$ & $\begin{array}{l}22.23 \\
(4.68) a\end{array}$ & & 263.67 & 439.45 \\
\hline $\mathrm{SE}(\mathrm{m})$ & 0.056 & 0.016 & 0.077 & & 11.924 & \\
\hline C.D. & 0.162 & 0.048 & 0.226 & & 34.804 & \\
\hline
\end{tabular}

*Figure in parenthesis is angular transformed values 
Table.3 Related yield and relative economics of various fungicidal treatments

\begin{tabular}{|c|c|c|c|c|c|}
\hline $\begin{array}{l}\text { Cercospora leaf } \\
\text { spot }\end{array}$ & $\begin{array}{l}\text { Yield } \\
\text { (kg/ha) }\end{array}$ & $\begin{array}{l}\text { Relative } \\
\text { cost } \\
\text { (Rs/ha) }\end{array}$ & $\begin{array}{l}\text { Relative } \\
\text { yield }(\mathrm{kg})\end{array}$ & $\begin{array}{l}\text { Relative } \\
\text { Return } \\
\text { (Rs/ha) }\end{array}$ & $\begin{array}{l}\text { Relative } \\
\text { net Profit } \\
\text { (Rs/ha) }\end{array}$ \\
\hline Control & 439.45 & & & & \\
\hline $\begin{array}{l}\mathrm{T}_{1} \\
\text { [Azoxystrobin + } \\
\text { Difenconazole } \\
\text { (Single } \\
\text { application)] }\end{array}$ & 947.22 & 4165 & 507.77 & 12694.25 & 8529.25 \\
\hline $\begin{array}{l}\mathrm{T}_{2} \\
\text { [Azoxystrobin + } \\
\text { Difenconazole } \\
\text { (Double } \\
\text { application)] }\end{array}$ & 1080.00 & 8330 & 640.55 & 16013.75 & 7683.75 \\
\hline $\begin{array}{l}\mathrm{T}_{\mathbf{3}} \\
\text { [Pyraclostrobin } \\
+ \text { Tebuconazole } \\
\text { (Single } \\
\text { application)] }\end{array}$ & 1285.00 & 1428 & 845.55 & 21138.75 & 19710.75 \\
\hline $\begin{array}{l}\mathrm{T}_{4} \\
\text { [Pyraclostrobin } \\
+ \text { Tebuconazole } \\
\text { (Double } \\
\text { application)] }\end{array}$ & 1650.55 & 2856 & 1211.1 & 30277.5 & 27421.5 \\
\hline $\begin{array}{l}\mathrm{T}_{5} \\
\text { [Carbendazim } \\
\text { (Double } \\
\text { application)] }\end{array}$ & 888.62 & 1820 & 439.45 & 10986.25 & 9166.25 \\
\hline $\begin{array}{l}\mathrm{T}_{6} \\
\text { [Chlorothalonil } \\
\text { (Double } \\
\text { application)] }\end{array}$ & 736.40 & 2410.8 & 296.55 & 7413.75 & 5002.95 \\
\hline
\end{tabular}

Disease Severity Scale

\begin{tabular}{|c|}
\hline Category \\
\hline $\mathbf{0}$ \\
\hline $\mathbf{1}$ \\
\hline $\mathbf{3}$ \\
\hline $\mathbf{5}$ \\
\hline $\mathbf{7}$ \\
\hline $\mathbf{9}$ \\
\hline
\end{tabular}

\begin{tabular}{|c|c|}
\hline Description \\
\hline No infection \\
\hline$<1 \%$ of area infected \\
\hline $1-10 \%$ of area infected \\
\hline $11-25 \%$ of area infected \\
\hline $26-50 \%$ of area infected \\
\hline More than $50 \%$ of area infected \\
\hline
\end{tabular}


Fig.1 Disease severity of Cercospora leaf spot on chilli

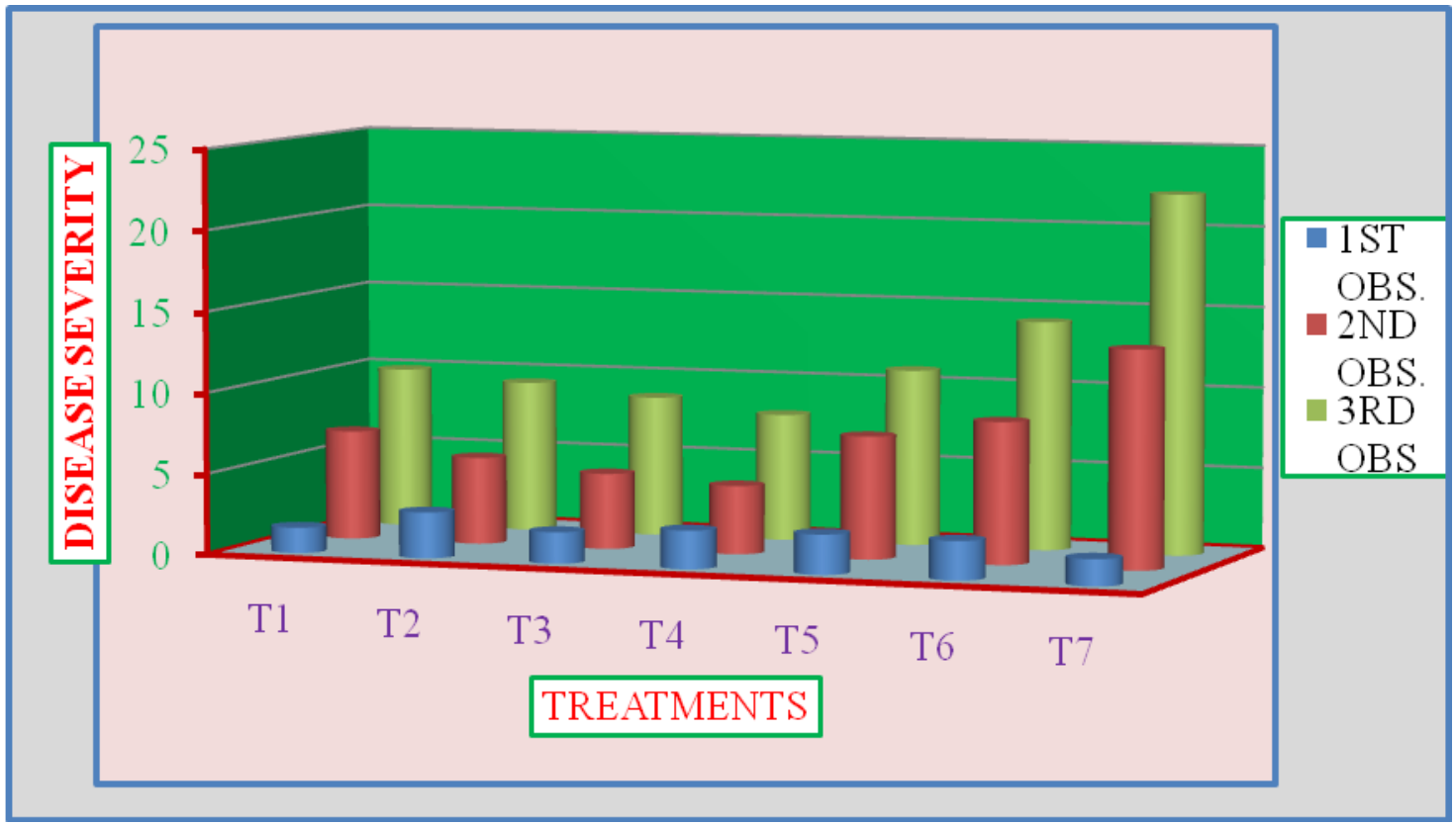

Fig.2 Disease control of Cercospora leaf spot of chilli

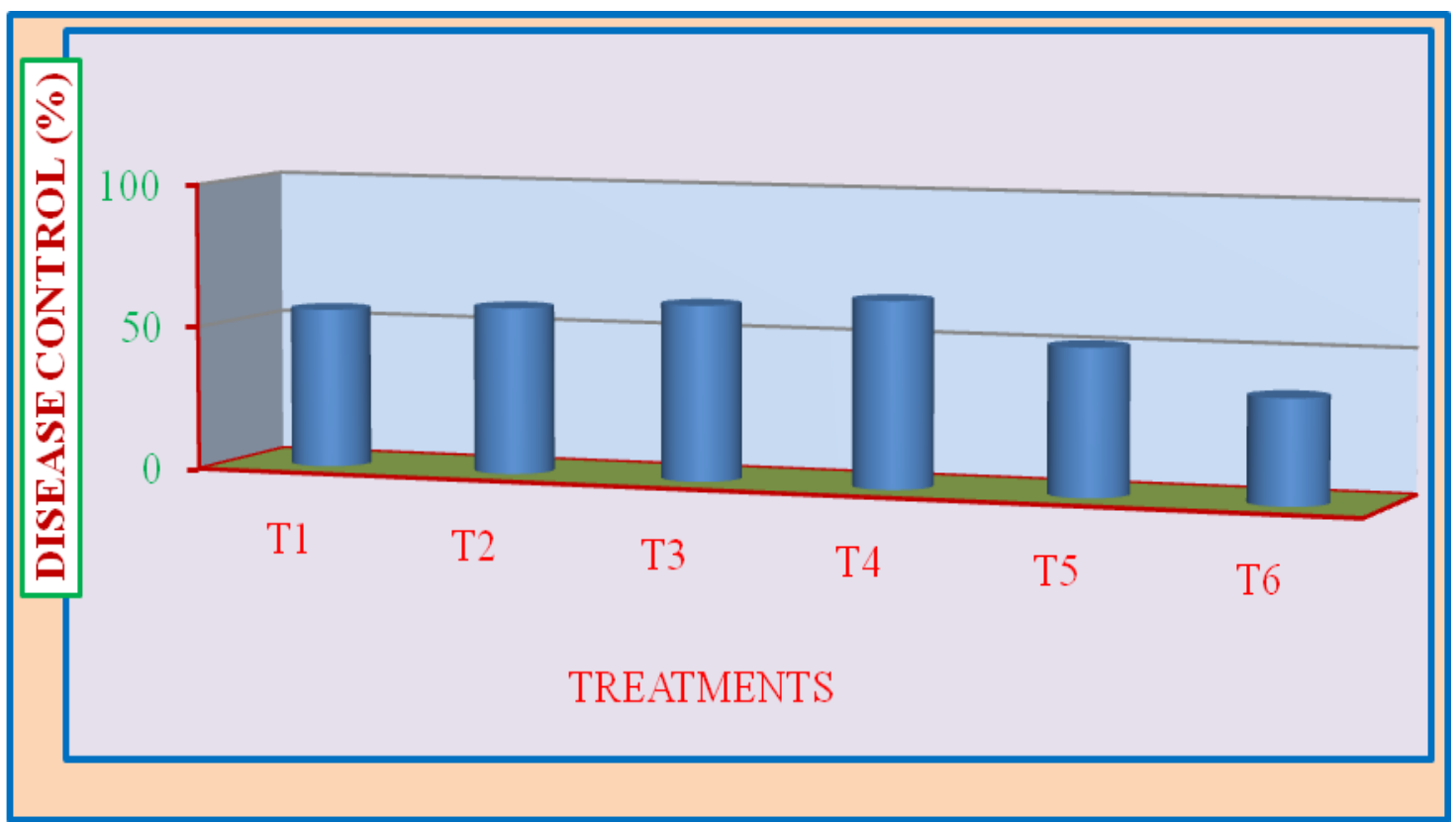

A good control of Cercospora leaf spot was found with combined formulation of Tebuconazole (Culbreath et al., 2008). Gastelum et al., (2007) reported that three spray application at weekly intervals of
Tebuconazole, Trifloxystrobin, Chlorothalonil provided an excellent control of leaf spot (Table 3). These findings are in line with the present experiment, where strobilurin fungicides, triazoles fungicides and some 
other fungicides like Chorothalonil are tested and found effective against Cercospora leaf spot.

\section{Acknowledgements}

The authors are thankful to the Department of Plant Pathology, UBKV for providing the necessary chemicals without which it would have been impossible to conduct the experiment.

\section{References}

Anonymous. 2012. NHB Database. 2012. www.nhb.gov.in

Bosland, P.W. and Votaven, E.J. 2003. Peppers: Vegetable and Spice Capsicums. CAB International. England, U.K. pp. 233.

Culbreath, A.K., Stevenson, K.L. and Brenneman, T.B. 2008. Management of leaf spot diseases of peanut with Prothioconazole applied alone or in combination with Tebuconazole or Trifloxystrobin. Peanut Science. 35(2): 149-158.
Felix-Gastelum, R, Avila-Diaz, J.A., Valenzuela-Cota, B.O., TriguerosSalmeron, J.A. and Longoria-Espinoza, R.M. 2007. Identification and chemical control of the causal agents of leaf spot and powdery mildew of husk tomato (Physalis ixocarpa brot.) in northern Sinaloa and Mexico. Revista Mexicana de Fitopatologia. 25(1): 1-10.

Khan, A.A., Khan, R.U. and Singh, R. 2005. Management of Cercospora leaf spot and anthracnose diseases of mungbean by fungicides. Annals of Plant Protection Sciences. 13(2): 514-515.

Mckinney, H.H. 1923. A new system of grading plant diseases. J. Agric. Res. 26: 195-218.

Poulos. 1992. Problems and Progress of Chilli Pepper Production in the Tropics. In: Hock, C. B., Hong, L. W., Rejab, M., Syed, A. R. (Eds.), Proceedings of the conference on Chilli Pepper Production in the Tropics. Kaula lumpur, Malaysia. pp. 98-129.

Tong, N. and Boseland, P.W. 1999. Capsicum tovarii: A new member of the Capsicum complex. Euphytica. 109(2): 71-72.

\section{How to cite this article:}

Dharnendra Reang, S. Khalko and Roy, A. 2018. Formulation of Effective Chemical Management Strategy against Cercospora Leaf Spot Disease of Chilli. Int.J.Curr.Microbiol.App.Sci. 7(10): 1240-1245. doi: https://doi.org/10.20546/ijcmas.2018.710.139 